OPEN ACCESS

Edited by:

Feng Gao,

Tianjin University, China

Reviewed by:

Yonghui Zeng,

University of Southern Denmark,

Denmark

Michal Koblizek,

Institute of Microbiology of the Czech Academy of Sciences, Czech Republic

*Correspondence:

Qiang Zheng

zhengqiang@xmu.edu.cn;

Nianzhi Jiao

jiao@xmu.edu.cn

Specialty section: This article was submitted to Evolutionary and Genomic Microbiology,

a section of the journal

Frontiers in Microbiology

Received: 02 May 2016

Accepted: 08 June 2016

Published: 24 June 2016

Citation:

Zheng Q, Lin W, Liu Y, Chen C and Jiao N (2016) A Comparison of 14

Erythrobacter Genomes Provides Insights into the Genomic Divergence

and Scattered Distribution of

Phototrophs. Front. Microbiol. 7:984.

doi: 10.3389/fmicb.2016.00984

\section{A Comparison of 14 Erythrobacter Genomes Provides Insights into the Genomic Divergence and Scattered Distribution of Phototrophs}

\author{
Qiang Zheng ${ }^{1 *}$, Wenxin Lin ${ }^{1}$, Yanting Liu ${ }^{1}$, Chang Chen ${ }^{2,3}$ and Nianzhi Jiao ${ }^{1 *}$ \\ ${ }^{1}$ State Key Laboratory of Marine Environmental Science, Institute of Marine Microbes and Ecospheres, Xiamen University, \\ Xiamen, China, ${ }^{2}$ CAS Key Laboratory of Tropical Marine Bio-resources and Ecology, South China Sea Institute of \\ Oceanology, Chinese Academy of Sciences, Guangzhou, China, ${ }^{3}$ Xisha Deep Sea Marine Environment Observation and \\ Research Station, South China Sea Institute of Oceanology, Chinese Academy of Sciences, Sansha, China
}

Aerobic anoxygenic phototrophic bacteria (AAPB) are bacteriochlorophyll a (Bchl a)-containing microbial functional population. Erythrobacter is the first genus that was identified to contain AAPB species. Here, we compared 14 Erythrobacter genomes: seven phototrophic strains and seven non- phototrophic strains. Interestingly, AAPB strains are scattered in this genus based on their phylogenetic relationships. All 14 strains could be clustered into three groups based on phylo-genomic analysis, average genomic nucleotide identity and the phylogeny of signature genes (16S rRNA and virB4 genes). The AAPB strains were distributed in three groups, and gain and loss of phototrophic genes co-occurred in the evolutionary history of the genus Erythrobacter. The organization and structure of photosynthesis gene clusters (PGCs) in seven AAPB genomes displayed high synteny of major regions except for few insertions. The 14 Erythrobacter genomes had a large range of genome sizes, from 2.72 to $3.60 \mathrm{M}$, and the sizes of the core and pan- genomes were 1231 and 8170 orthologous clusters, respectively. Integrative and conjugative elements (ICEs) were frequently identified in genomes we studied, which might play significant roles in shaping or contributing to the pan-genome of Erythrobacter. Our findings suggest the ongoing evolutionary divergence of Erythrobacter genomes and the scattered distribution characteristic of PGC.

Keywords: Erythrobacter, integrative and conjunctive element, photosynthesis gene cluster, aerobic anoxygenic phototrophic bacteria, comparative genomics

\section{INTRODUCTION}

Aerobic anoxygenic photoheterotrophic bacteria (AAPB) are bacteriochlorophyll $a$ (Bchl $a)$ containing and obligate aerobic bacteria, and they are widely distributed in the euphotic zone of the ocean (Kolber et al., 2001; Yurkov and Csotonyi, 2009). These phototrophic microorganisms account for $1-15 \%$ of the total bacteria in the upper ocean, and the BChl $a$-based phototrophy could reduce respiratory organic carbon consumption of $\sim 2.4-5.4 \%$ of marine primary productions (Jiao et al., 2010; Ritchie and Johnson, 2012; Ferrera et al., 2014; Stegman et al., 2014). Thus, AAPB could potentially play significant roles in carbon and energy cycling in the ocean (Kolber et al., 2001; Jiao et al., 2007; Kobližek et al., 2007). 
Currently, the known marine AAPB isolates are classified into Proteobacteria, including Alpha- and Gammaproteobacteria. Alphaproteobacterial AAPB mainly comprises the Roseobacter clade (e.g., genera Roseobacter, Dinoroseobacter, Roseivivax, Roseovairus, and Roseibacterium) and the Eryth-Citro clade, including the genera Erythrobacter and Citromicrobium (Béjà et al., 2002; Yutin et al., 2007; Zheng et al., 2011). Most cultured marine Gammaproteobacterial AAPB belong to the clade NOR5/OM60 (Cho et al., 2007; Fuchs et al., 2007; Spring et al., 2009).

AAPB possess a highly conserved photosynthesis gene cluster (PGC) including bch, crt, puf, puh, and some regulatory genes (Blankenship, 1992; Beatty, 1995; Swingley et al., 2009; Zheng et al., 2011). For the evolutionary history of AAPB, both gain and loss of PGC were detected as revealed by comparison of AAPB and closely related nonAAPB genomes. Gemmatimonas sp. AP64, which belongs to phylum Gemmatinonadetes, might obtain its PGC from purple phototrophic bacteria (Alplaproteobacteria) by horizontal gene transfer (Zeng et al., 2014). Citromicrobium sp. JLT1363, which is classified into Alpha-IV subclade, lost the PGC from the long-term evolutionary history and became a completely heterotrophic bacterium (Zheng et al., 2012). Previous studies have shown that the AAPB in the ErythCitro clade contain unique carotenoid biosynthetic pathways and pigments compared with the Roseobacter clade (Kobližek et al., 2003; Zheng et al., 2011, 2013). The structure of the photosynthesis gene cluster (PGC) in the Eryth-Citro clade was the shortest and simplest among all known $\mathrm{AAPB}$, and no light-harvesting complex II (LH II) genes were found in their genomes (Zheng et al., 2011, 2013). The obligate aerobic characteristics and unique PGC structure suggest that these AAPB in the Eryth-Citro diverged long time ago with AAPB belonging to Roseobacter clade (Zheng et al., 2012, 2013, 2014). However, few studies have been focused on the evolution of AAPB in Erythrobacter genus to date, although a great number of strains have been sequenced in this genus.

The genus Erythrobacter was established following the isolation and identification in 1982 of the first AAPB strain Erythrobacter longus DSM 6997 (Shiba and Simidu, 1982). Later, the second AAPB species belonging to Erythrobacter genus, E. litoralis DSM 8509, was identified by Yurkov et al. (1994). Since then, a variety of strains belonging to this genus have been recognized from diverse habitats (Kobližzek et al., 2003; Zheng et al., 2014; Lei et al., 2015; Zhuang et al., 2015), and some of them lack Bchl a (Anderson et al., 2009; Oh et al., 2009; Wei et al., 2013). By the end of 2015, 18 species and dozens of strains had been isolated and identified in the genus Erythrobacter (Tonon et al., 2014; Zheng et al., 2014; Lei et al., 2015; Zhuang et al., 2015). Interestingly, photoheterotrophic bacteria did not cluster together within one genus based on their phylogenetic relationship. The aim of this study is trying to address (1) the evolutionary divergence of their genomes and (2) the distribution pattern and evolution of photosynthesis gene cluster in the genus Erythrobacter.

\section{MATERIALS AND METHODS}

\section{Bacterial Strains}

Fourteen Erythrobacter spp. strains were used for bioinformatic analyses. Five of these were sequenced by our lab: Erythrobacter longus DSM 6997, Erythrobacter litoralis DSM 8509, Erythrobacter sp. JL475, Erythrobacter sp. YT30, and Erythrobacter sp. AP23. Erythrobacter longus DSM 6997 and Erythrobacter litoralis DSM 8509 were purchased from the DSMZ culture collections. Strains JL475, YT30, and AP23 were isolated from the South China Sea using extinction dilution method on rich organic medium (Yurkov et al., 1999) and maintained in the laboratory.

The other nine Erythrobacter genomes were collected from the National Center for Biotechnology Information (NCBI), and their GenBank accession numbers follow: Erythrobacter sp. NAP1 (AAMW00000000; Kobližek et al., 2011), Erythrobacter marinus HWDM-33 (LBHU00000000; Jung et al., 2012), Erythrobacter litoralis HTCC2594 (NC007722; Oh et al., 2009), Erythrobacter sp. s21-N3 (CP011310; Zhuang et al., 2015), Erythrobacter sp. SD-21 (ABCG00000000; Anderson et al., 2009), Erythrobacter gangjinensis K7-2 (LBHC00000000; Lee et al., 2010), Erythrobacter vulgaris O1 (CCSI00000000; Yaakop et al., 2015), Erythrobacter citreus LAMA 915 (JYNE00000000), and Erythrobacter sp. KA37 (LBHB00000000; Lei et al., 2015).

\section{Genome Sequencing, Assembly, and Annotation}

Three draft genomes of strains DSM 6997, DSM 8509, and JL475 were obtained using Illumina HiSeq sequencing technology in Chinese National Human Genome Center at Shanghai. Two libraries with average sizes of 150 and $500 \mathrm{bp}$ were constructed using the TruSeq TM DNA Library Prep Kit (Illumina, USA). Paired-end reads of an average length of $100 \mathrm{bp}$ were assembled using Velvet software (V1.2.03) (Zerbino and Birney, 2008), and a total read size of $\sim 2.5 \mathrm{Gbp}$ for each strain was obtained.

The genomes of strains YT30 and AP23 were obtained using the Illumina MiSeq system in Shanghai Personal Biotechnology Limited Company. Paired-end reads of an average length of 250 bp were assembled using Velvet software (v2.8; Zerbino and Birney, 2008). The sequencing coverage was $\sim 300 \mathrm{X}$ for strains AP23 and YT30.

The prediction and annotation of open reading frames (ORFs) were performed with Rapid Annotation using Subsystem Technology (RAST; Aziz et al., 2008). The rRNA and tRNA identification was performed with RNAmmer 1.2 software (Lagesen et al., 2007) and tRNAscan-SE (v1.21; Lowe and Eddy, 1997), respectively.

The genomic average nucleotide identity (ANI) was calculated with the JSpecies Web online service (http://jspecies.ribohost. com/jspeciesws; Richter and Rosselló-Móra, 2009).

\section{Core Genome and Pan-Genome Analyses}

Orthologous clusters (OCs) were analyzed using OrthoMCL, and all protein sequences from the 14 genomes were grouped based on the sequence similarity $\left(E<10^{-5},>50 \%\right.$ coverage; Li et al., 2003). All genes from fourteen Erythrobacter genomes 
were selected to calculate the core and pan-genomes. The sizes of core and pan-genomes were calculated based on the number of genomes involved in the analysis (Tettelin et al., 2005).

\section{Phylogenetic Analysis}

The sequences were aligned using Clustal X, and phylogenetic trees were constructed using the neighbor-joining and maximum likelihood algorithms of MEGA 6 software (Tamura et al., 2013). The phylogenetic trees were supported by bootstrap for the resampling test with 1000 and 100 replicates for the neighborjoining and maximum likelihood algorithms, respectively.

\section{Accession Numbers}

The whole-genome sequences of strains DSM 6997, DSM8509, JL475, AP23, and YT30 are available under GenBank accession numbers JMIW00000000, JMIX00000000, JMIV00000000, LNBY00000000, and LMAF00000000, respectively.

\section{RESULTS AND DISCUSSION \\ General Features of the Erythrobacter Strains}

Fourteen strains isolated from diverse aquatic environments were used for comparative genome analyses (Table 1). Seven of them containing complete PGC were AAPB strains, including Erythrobacter sp. NAP1, E. longus DSM 6997, E. litoralis DSM 8509, and Erythrobacter sp. JL475, Erythrobacter sp. AP23, Erythrobacter sp. YT30 and E. marinus HWDM-33. The other seven strains containing no PGC were non-AAPB. All shared more than 94\% $16 \mathrm{~S}$ rRNA sequence similarity. However, the nucleotide identities among the seven pufM sequences were $<80 \%$.

From the phylogenetic trees based on the 16S rRNA gene, 14 Erythrobacter strains could be clustered into three groups: strains DSM 6997, NAP1, JL475, DSM 8509, YT30, and HTCC2594 formed one group (Group I), strains SD-21, O1, LAMA 915, and AP23 formed a second group (Group II), and the other four strains formed a third group (Group III; Figure 1A). The AAPB strains were scattered in three groups. The $16 \mathrm{~S}$ rRNA sequence identities within each group (I and II) were more than 97 and $98 \%$, respectively. The strains belonging to Group III showed much lower identities than the other two groups, ranging from 94 to $97 \%$. Seven AAPB strains were grouped into three clades. The scattered distribution pattern of phototrophs was also found in Rosoebacter clade (Wagner-Döbler and Biebl, 2006; Koblížek et al., 2013). That indicates the evolution and distribution pattern of PGCs might be prevalent in Alphaproteobacteria.

\section{Genomic Information for the Erythrobacter Strains}

The genomic size varied from 2.72 M (E. gangjinensis K7-2) to 3.60 M (E. longus 6997), and the total GC content ranged from 57 to $65 \%$ (Table 1). The number of genes is consistent with the genomic size. The number of structural RNAs ranged from 42 to 46 . The average genome coverage for newly sequenced five strains, JL475, DSM 6997, DSM 8509, YT30, and AP23 were 780X, 680X, 780X, 299X, and 298X, respectively (Table 1).

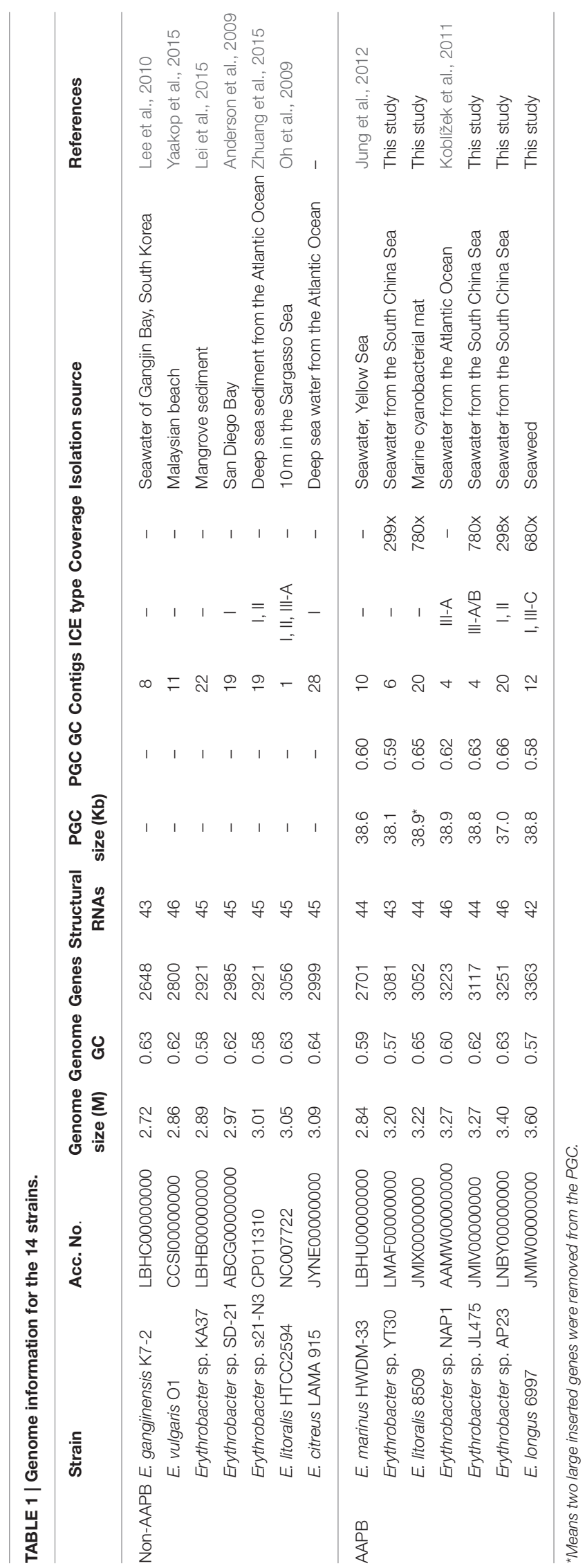




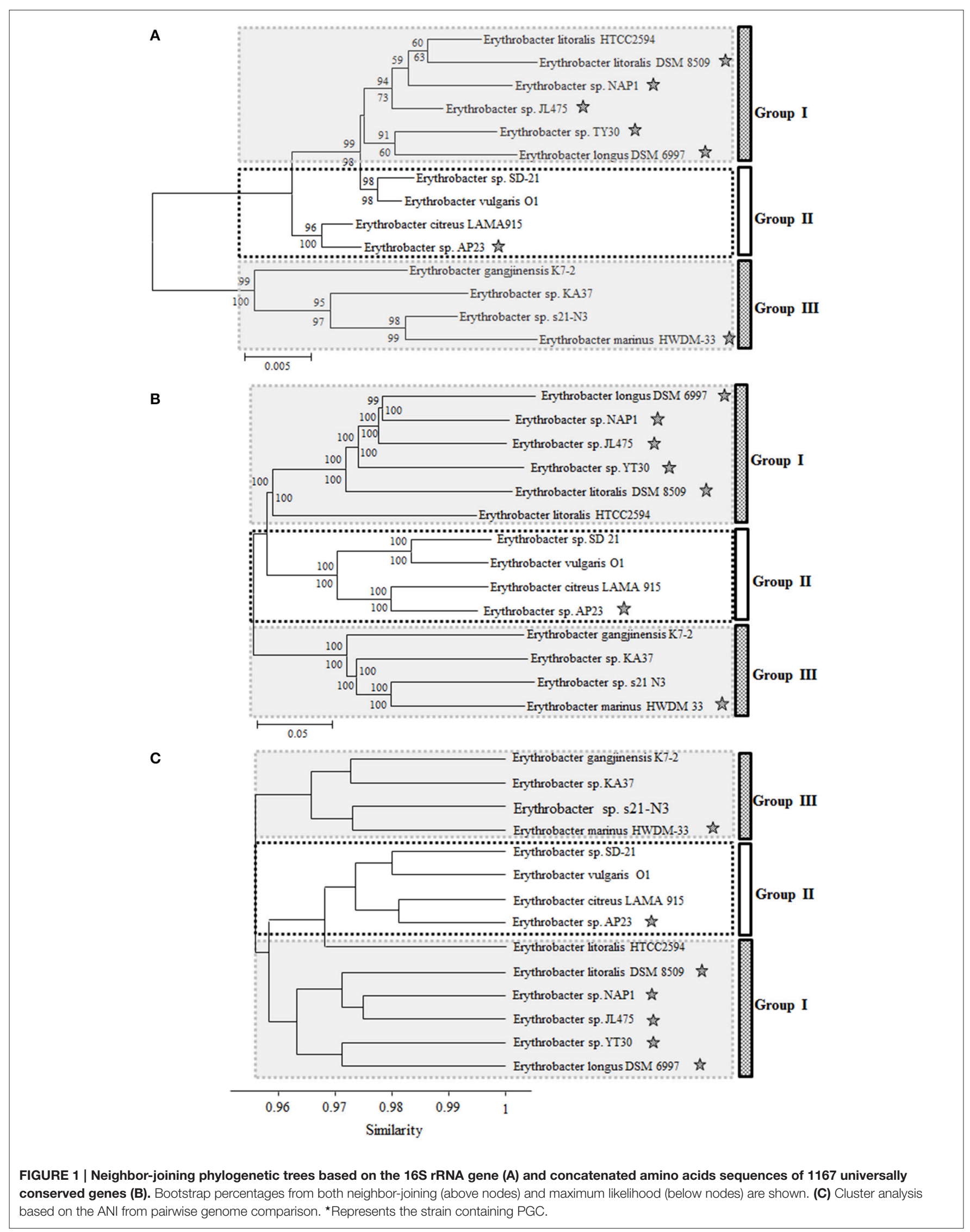


The phylogenetic tree based on the concatenated amino acids sequences of 1167 universally conserved genes among these 14 investigated Erythrobacter genomes was consistent with $16 \mathrm{~S}$ rRNA phylogeny and also formed three groups (I, II, and III) (Figure 1B). The genomic sizes of AAPB in Group I (3.20 3.60 $\mathrm{M}$ vs. $3.05 \mathrm{M})$ and II (3.40 M vs. $2.86 \sim 3.09 \mathrm{M}$ ) were significantly larger than non-AAPB in the same groups. All four strains in Group III (avg. $\sim 2.86 \mathrm{M}$ ) showed relatively small genomic sizes compared with the other two groups (avg. $\sim 3.19 \mathrm{M}$ ).

\section{The Genomic Average Nucleotide Identity}

The Average Nucleotide Identity (ANI) shared between Erythrobacter genome pairs ranged from 68.78 to $81.34 \%$ (Table S1). Taking into account the proposed cut-off of the ANI between genome pairs for a species boundary of 95-96\% (Richter and Rosselló-Móra, 2009), all analyzed 14 Erythrobacter strains were separated species; however, some of them shared high $16 \mathrm{~S}$ rRNA sequence identities. The low genomic percentage (ranging from 33.60 to $70.81 \%$ ) involved in pairwise comparisons indicated that they diverged a long time ago.

The genome pairs in each group showed relatively high ANI and genomic percentages involved in pairwise comparisons. The genome pairs in Group III shared $72.77-74.58 \%$ ANI, and the genomic percentages involved in pairwise comparisons ranged from 48.05 to $59.81 \%$ (Table S1). While the ANI values between genome pairs (one from Group III and the other from Group I or II) were $68.78-70.40 \%$ and $70.16-70.96 \%$, respectively, the genomic percentages involved in pairwise comparisons were $36.31-41.32 \%$ and $37.05-44.42 \%$, respectively (Table S1).

The ANI and genomic percentages involved in pairwise comparisons (Group II vs. Group I or III; Group I vs. Group II or III) had similar characteristics to Group III vs. Group I or II. In consistency with the $16 \mathrm{~S}$ rRNA phylogenetic tree, strain HTCC2594 was clustered into Group I in the whole genome tree. However, strain HTCC2594 shared a higher ANI and involved a larger genomic percentage according to the pairwise comparison with the genomes in Group II (Figure 1C).

\section{The Pan- and Core Genomes of the Erythrobacter Strains}

The Erythrobacter pan-genome for 14 sequence strains comprised 8170 predicted orthologous clusters (OCs), and the core genome contained 1231 OCs (Figure S1). The cumulative length of all core genes was approximately 1.20 Mbp, which covered only $33-45 \%$ of the genome content. The flexible genome comprises 6939 OCs including 3815 unique OCs and 3124 shared by more than one strain but not all strains. The number of genes for the core genome appeared to reach a plateau, whereas the genes for the pan-genome increased with genome number (Figure S1).

The core genome is mainly involved in central metabolism and housekeeping functions, from the Glycolysis to the TCA cycle. Approximately $94.7 \%(16,328 / 17,234)$ of the predicted core genes were assigned to COG functional categories. The predicted core genes include a relatively high percentage of genes assigned to the following COG categories: translation, ribosomal structure and biogenesis $(\mathrm{J})$, general function prediction only $(\mathrm{R})$, amino acid transport, and metabolism (E), energy production and conversion (C), and unknown function (S) (Figure 2A). Due to a larger fraction of putative or hypothetical genes, only $74.1 \%(14,622 / 19,723)$ of flexible genes were assigned to COG functional categories. Compared with the core genes, flexible genes contain an overrepresentation of genes assigned to the following COG categories: cell motility $(\mathrm{N})$, secondary metabolites biosynthesis, inorganic ion transport and metabolism (P), lipid transport and metabolism (I; Figure 2B). Most of the flexible genes were sourced from the genetic island regions.

Genomic rearrangements and highly variable genetic islands were prevalent features as comparison of gene composition and arrangement in these 14 strains. Integrase and ICE were frequently found in their chromosomes, which seemed to be an important force in shaping their genomic composition and evolutionary divergence.

\section{Photosynthesis Gene Cluster}

The sizes of the PGCs ranged from 37.0 to $38.9 \mathrm{~kb}$, which represented $\sim 1.08-1.36 \%$ of the genomes. The GC contents of the PGCs varied from 58 to $66 \%$, which was similar to the total GC contents of the corresponding genomes (Table 1). The PGC organization in the Erythrobacter genus comprises two conserved subclusters, bchIDO-crtCDF-bchCXYZ-pufBALM and bchFNBHLM-lhaA-puhABC-acsF-hyp-puhE-hemA (Figure 3). The PGC arrangement in this genus belonged to type III (forward crtF-bchCXYZ-puf plus forward bchFNBHLM-LhaApuh; Zheng et al., 2011, 2013). All PGCs were almost identical in terms of gene arrangement and composition (Figure 3). An inserted gene was involved in the outer membrane protein and the pseudoazurin gene among the PGCs belonging to E. longus DSM 6997 and Erythrobacter sp. JL475, respectively.

Five AAPB strains belonging to Group I shared the same upstream and the similar downstream genes of PGCs (Table S2), and their PGCs clustered together (Figure 4). This indicated that these five PGCs diverged from a common ancestor. Upstream of these five PGCs, there was a conserved gene cluster in the order of type IV secretion system (T4SS), TonB-dependent transporter and iron $\mathrm{ABC}$ transporter. Downstream, the PGCs were flanked by permease, toxin secretion $\mathrm{ABC}$ transporter, (outer) membrane protein, and isoquinoline oxidoreductase. The genome of strain HTCC2594 showed genomic recombination close to the corresponding position in the five other Group I AAPB strains. Here, two explanations were proposed to account for the evolution of PGC in Group I: the first is that an HTCC 2594-related strain acquired PGC via horizontal gene transfer a long time ago and then diverged; the other is that some photoheterotrophic ancestors lost the PGC and thus became heterotrophic.

The size of the PGC in strain AP23, which had the highest GC content $(66 \%)$, was the shortest $(37.0 \mathrm{~kb})$ among all known AAPB (Table 1). It had the same gene composition and organization as the AAPB in Group I. However, it displayed a unique flanking gene compared with the AAPB strains in Groups I and III. Integrase, which was inserted after tRNA-Pro-GGG, 


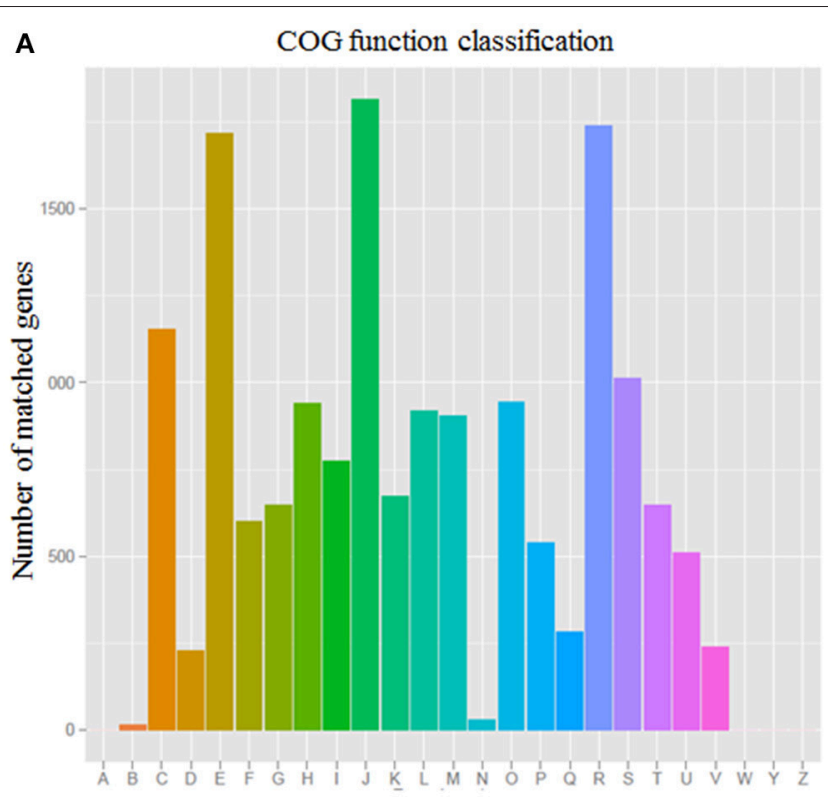

B

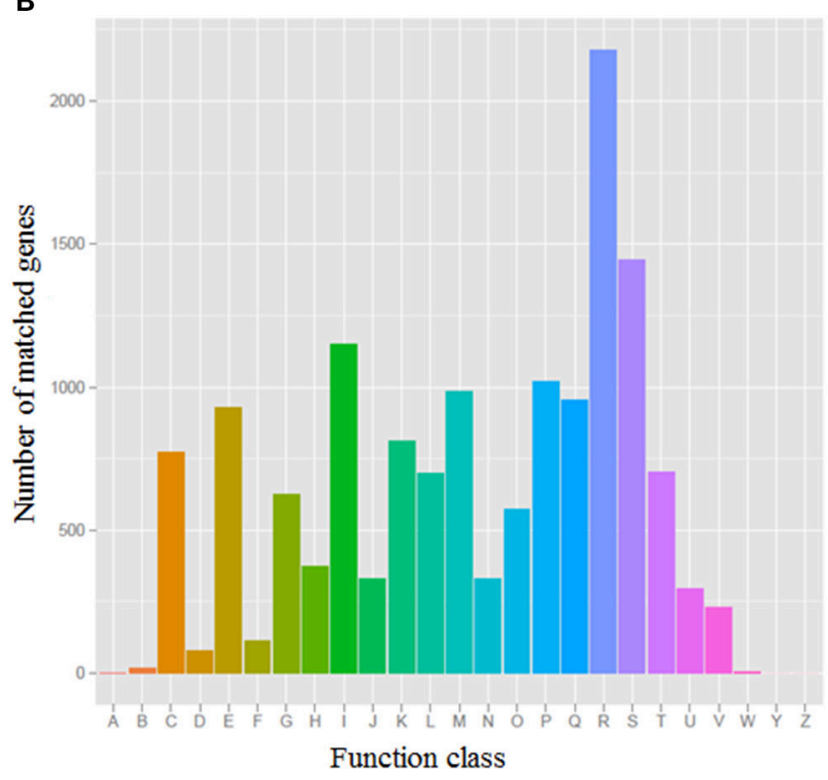

A:RNA processing and modification

B:Chromatin structure and dynamics

C:Energy production and conversion

D:Cell cycle control, cell division, chromosome partitioning

E:Amino acid transport and metabolism

F:Nucleotide transport and metabolism

G:Carbohydrate transport and metabolism

$\mathrm{H}$ :Coenzyme transport and metabolism

I:Lipid transport and metabolism

$\mathrm{J}$ :Translation, ribosomal structure and biogenesis

K: Transcription

L:Replication, recombination and repair

M:Cell wall/membrane/envelope biogenesis

$\mathrm{N}$ :Cell motility

O:Posttranslational modification, protein turnover, chaperones

P:Inorganic ion transport and metabolism

Q:Secondary metabolites biosynthesis, transport and catabolism

$R:$ General function prediction only

S:Function unknown

T:Signal transduction mechanisms

U:Intracellular trafficking, secretion, and vesicular transport

V:Defense mechanisms

W:Extracellular structures

Y:Nuclear structure

Z:Cytoskeleton

FIGURE 2 | COG function classification of core (A) and flexible (B) genes from 14 Erythrobacter genomes.

was found in front of the PGC in strain AP23, and its genes upstream and downstream of PGC were found together in other genomes. The phylogeny indicated that its closest relative was the Citromicrobial strains (Figure 4). Strains LAMA 915 and AP23 shared $99.5 \%$ identity at the level of the 16S rRNA gene sequence, and the genome sequence of strain LAMA 915 contained no PGC. That suggested the PGC in strain AP23 was acquired by horizontal gene transfer. In the environment, bacteria are much easier to accept foreign genes from closely related strains (Jain et al., 1999; Koonin et al., 2001). However, it would be difficult to find the HGT event based only on the phylogenetic relationship if that happens.
The PGC in strain HWDM-33 was located after the T4SS. However, there were two copies of T4SS in strains HWDM33, KA37, SD-21, and YT30, which usually mediates interbacterial DNA transfer, and secretion of virulence factors into target cells (Yeo and Waksman, 2004; Alvarez-Martinez and Christie, 2009).The first copy found in all strains coevolved with their genomes based on the phylogeny of the virB4 gene (Figure S2), and the second copy only detected in four strains (HWDM-33, KA37, SD-21, and YT30) appeared to be obtained by HGT. The type IV secretion system was frequently found in Alphaproteobacteria, and it has been predicted to play roles in natural transformation as a mechanism for gene exchange 


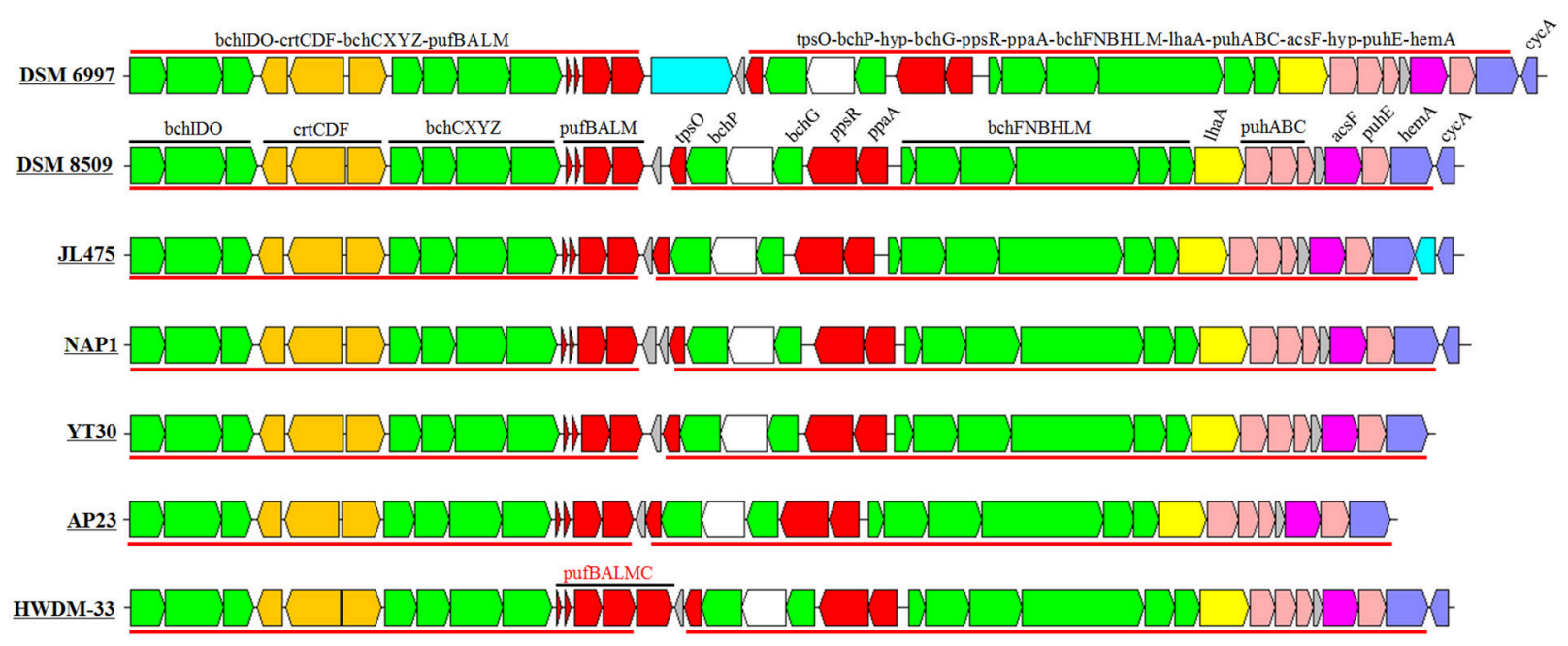

FIGURE 3 | Structure and arrangement of PGCs in Erythrobacter. Green, bch genes; red, puf and regulator genes; pink, puh genes; orange, crt genes; blue, hem and cyc gene; yellow, IhaA gene; blank, uncertain or unrelated genes; and gray, hypothetical protein. The horizontal arrows represent putative transcripts.

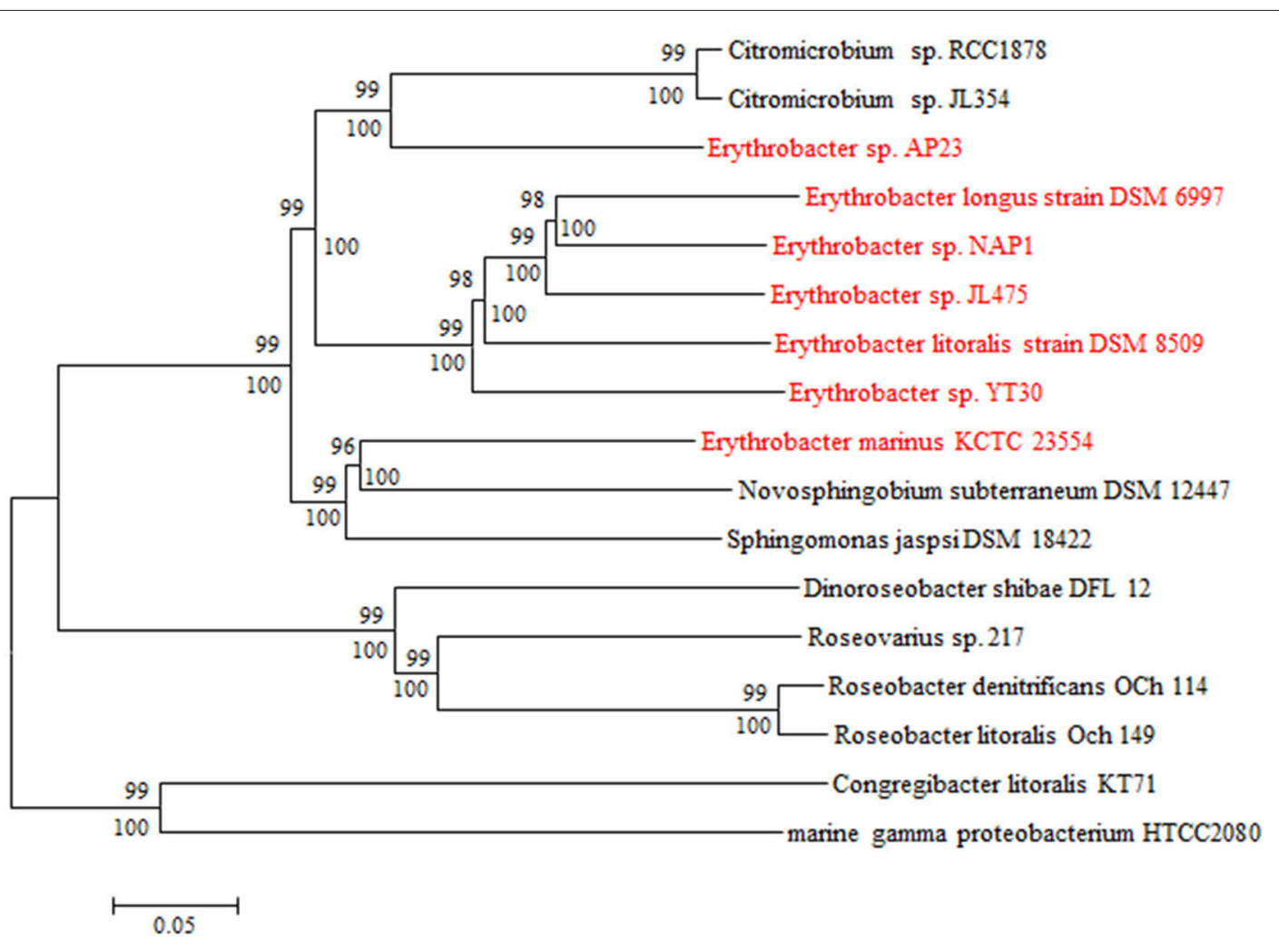

FIGURE 4 | Neighbor joining phylogenetic analysis of concatenated amino acids sequences of 27 universally conserved genes (9415 positions) in PGCs from GenBank database. The core genes are bchBCDFGHILMNOPXYZ-crtCF-pufABLM-IhaA-puhABCE-ascF. Bar, 0.1 substitutions per amino acids position.

(Hubber et al., 2004; Aylward et al., 2013). In addition, the reaction center (RC) consists of three pufLMC genes in strain HWDM-33 instead of pufLM, which existed in six other AAPB strains in this genus. Thus, we speculated that the type IV secretion of the T-DNA complex (Table S3) might mediate the HGT of the PGC in strain HWDM-33.

There are two known types of RC in AAPB: one has a tightly bound subunit of a c-type cytochrome ( $p u f L M-p u f C$ ) that 
acts as the direct electron donor to a photo-oxidized special pair of bacteriochlorophylls; the other type accepts electrons directly from water-soluble electron carriers such as cytochrome c2 (cycA; Nitschke and Dracheva, 1995). In all Erythrobacter AAPB genomes except strain HWDM-33, the pufC gene was absent. The recent study suggested that the pufC gene is not essential for photosynthetic growth and that it might accelerate the re-reduction of the primary electron donor (Verméglio et al., 2012).

A previous study showed that the main difference among PGCs was the genes encoding the carotenoid biosynthetic pathway. The complete set of crt genes identified in Rba. capsulatus was crtAIBKCDEFJ (Zheng et al., 2011). A slightly reduced set of genes was found in some Roseobacter and NOR5 species. In comparison, only crtCDF existed in the PGCs of

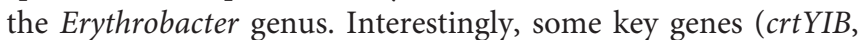
$\operatorname{crt} W Z$, and $c r t G$ ) for pigment biosynthesis were not organized in the PGCs in Erythrobacter, and instead they are scattered in the chromosome. The $c r t$ genes ( $c r t Y I B$ and $c r t W Z$ ) were observed in all seven analyzed non-AAPB strains.

\section{Integrative and Conjugative Elements}

The genome size of all AAPB except strain HWDM-33 is significantly larger than any of non-AAPB strains. AAPB or non-AAPB containing ICE structures possess relatively large genome size compare to AAPB or non-AAPB without ICE respectively (Table 1). The large pan-genome size of Erythrobacter indicated that it had some capacity to obtain foreign genes, and these frequently found ICEs contributed to the flexible genome and possibly to environmental adaptation.

ICEs are bacterial self-transmissible mobile genetic elements that can integrate into and be excised from the chromosome (Böltner et al., 2002; Burrus et al., 2006). ICEs possess features of both temperate bacteriophages (the front part) and conjugative plasmids (the latter part; Figure 5; Burrus et al., 2006; Wozniak et al., 2009). ICEs have recently been shown to contain several intergenic hotspots where a variety of new traits and adaptive functions can be obtained, including resistance to antimicrobial compounds, heavy metals or bacteriophage infection (Wozniak et al., 2009). The core regions display conservative gene synteny with fewer insertions or deletions, which suggests their importance for ICE self-transfer (Gaillard et al., 2010; Miyazaki et al., 2015). ICEs have been reported to be the most abundant conjugative elements in bacteria (Guglielmini et al., 2011; PoulinLaprade et al., 2015), and they are a major driving force of bacterial genome plasticity and evolution (Böltner et al., 2002; Burrus et al., 2006).

Eight of fourteen genomes were found to contain 14 ICEs. Strain HTCC2594 possessed three ICEs; strains s21-N3, JL475, AP23, and DSM 6997 contained two ICEs; strains SD-21, LAMA915, and NAP1 had one ICE. Two ICEs in strain JL475 were combined together, and one of them lost part of its genes for phage functions. Therefore, there were a total of 13 integrases in the 14 ICEs.

All 14 ICEs, except for the incomplete one in strain JL475, were integrated into the host chromosome after a tRNA gene and could be grouped into three clades based on the different types of tRNA genes. Six ICEs were located after the tRNA-Leu-CAG gene; five ICEs were flanked by the tRNA-Met-CAT gene; three ICEs were integrated after the tRNA-Val-CAC gene.

ICEs identified a specific chromosome position (different tRNA) and then integrated into the chromosome in the $5^{\prime}-3^{\prime}$ or $3^{\prime}-5^{\prime}$ direction. The tRNA-Val-CAC gene is always found at the end of the ICEs, indicating that the ICE integrated into the chromosome in the $3^{\prime}-5^{\prime}$ direction. The other ICEs integrated into the chromosome in the $5^{\prime}-3^{\prime}$ direction, and the integrase was just after the tRNA-Met-CAT or tRNA-Leu-CAG. This suggested that different types of ICEs underwent site-specific and directionspecific insertions. Interestingly, two integration events occurred at the tRNA-Leu-CAG gene position in strains AP23 and s21-N3.

Two complete prophages were detected in strains KA37 and LAMA915. The prophage in strain KA37 encoded an integrase and was integrated into the host chromosome after the tRNATyr-GTA gene. This integrase was considered a reference and also added into the following analysis.

All integrases after four tRNAs (tRNA-Leu-CAG, tRNA-MetCAT, tRNA-Val-CAC, and tRNA-Tyr-GTA) in 14 genomes were collected for the phylogenetic analysis (Figure 6A). Generally, all integrases originating from the same tRNA gene were clustered together except the one in the prophage. These integrases from the ICEs formed three clades (I, II, and III) based on the three tRNA types (Figure 6A). Integrases, which were not from ICEs, also clustered with the corresponding tRNA clades. However, the integrase of the prophage was completely different from those found in other genomes at the tRNA-Tyr-GTA gene position

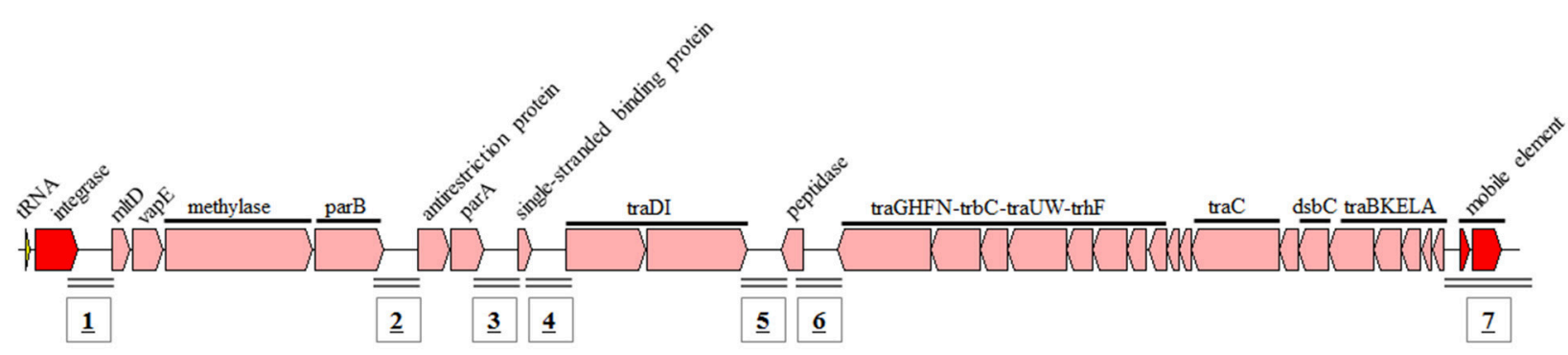

FIGURE 5 | Structure and composition of ICE. Seven hotspots (from No. 1 to 7) carrying exogenous genes were detected in the ICES. 

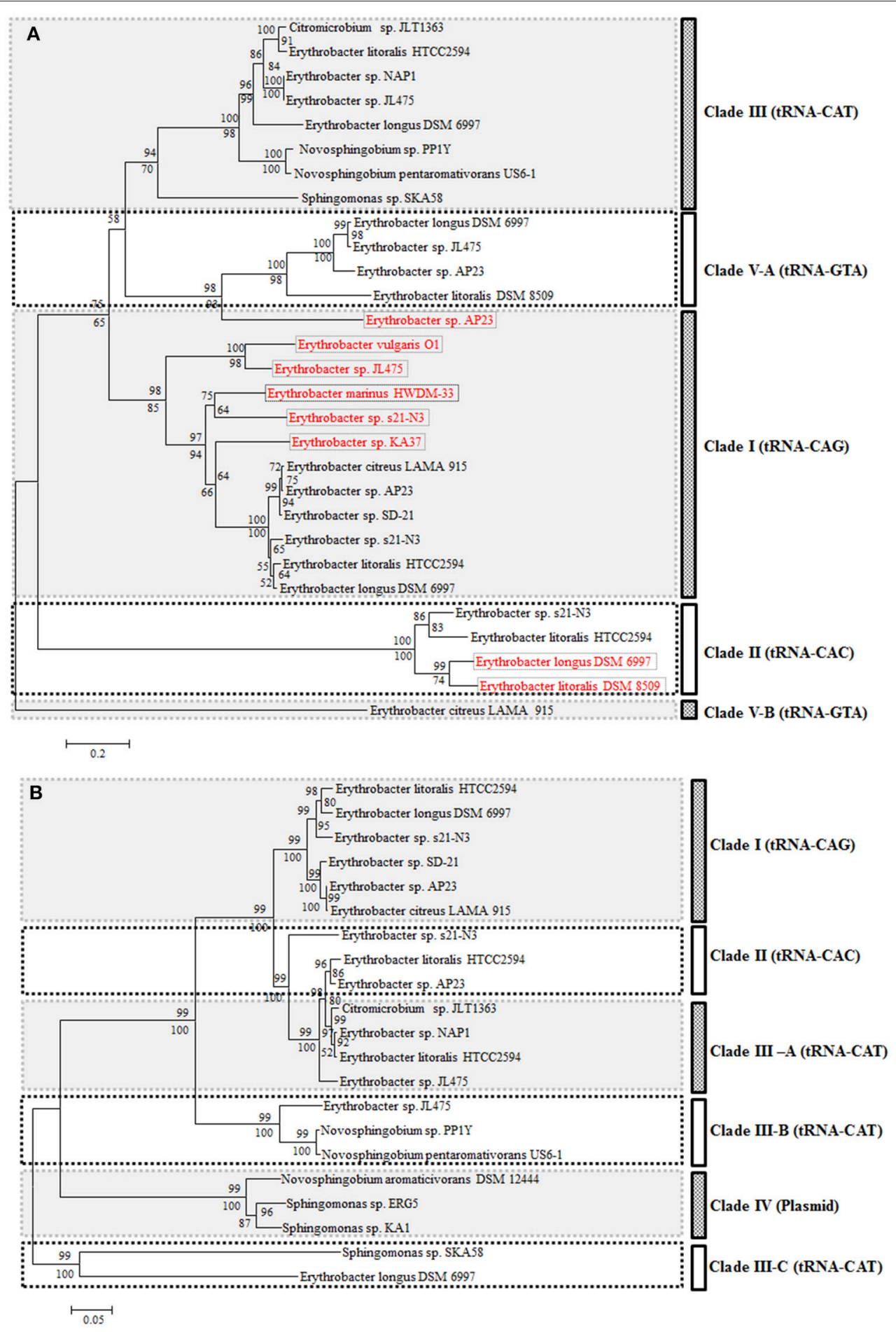

FIGURE 6 | Neighbor-joining phylogenetic trees based on Integrase (A, 430 amino acid positions) and TraC (B, 846 amino acid positions). Bootstrap percentages from both neighbor-joining (above nodes) and maximum likelihood (below nodes) are shown.

(Figure 6A). This suggested that different types of phages could integrate into the same position in the chromosome.

As seen from the comparison of the phylogeny based on the integrase and $\mathrm{TraC}$, the evolution of the two parts was not completely synchronous. The phylogeny based on the TraC showed more diversity than the integrase. However, these TraC sequences were clustered together and located at the same tRNA position (Figure 6B). Clade III could be grouped into 
three sub-clades based on the TraC sequences. All of the sequences from the ICEs were distinguished by plasmid origins (Figure 6B).

It seemed that the ICEs possessed incompatibility similar to plasmids. The ICEs from the same host fell into different phylogenetic clades. Two ICEs were located at the same position in strain JL475, but one was an incomplete or defective element. Furthermore, two of them fell into different sub-clades.

From the 14 ICEs, 7 potential hotspots carrying foreign genes were identified (Figure 5) and the inserted gene fragment ranged from $1-2 \mathrm{~kb}$ to $\sim 100 \mathrm{~kb}$. Overall, 33 and 34 mobile elements were found in 6 and 3 ICEs belonging to Clades I and II, respectively. Only three mobile elements were detected in 5 IECs belonging to Clade III, suggesting that the three types of ICEs displayed different capacities for gene exchange between the ICE and the host genome.

Members of ICEs in different clades were responsible for carrying different functional genes. The ICEs in clade I preferred to carry genes coding for a nucleotide-metabolism-related function, such as a complete type I restriction-modification system, type IIS restriction enzyme, DNA double-strand break repair protein, DEAD-box helicase-related protein, superfamily I/II DNA/RNA helicases, ribonucleotide reductase of class III, DNA methyltransferase, and so on. In addition, a complete respiratory nitrate reductase system was discovered in strain DSM 6997.

Members of the ICEs in clade III mainly carried heavy metal resistance genes, including lead, cadmium, zinc, mercury, nickel, cobalt, and arsenicals. An 18.5-kb DNA fragment involved in heavy metal resistance in the clade III ICE of strain HTCC 2594 (Positions 905,233 to 923,810) was identical to that found in Citromicrobium sp. JLT1363 (AEUE01000001, positions 368,467 to 387,044 ), which also was located in the clade III ICE. These two strains shared $94.8 \%$ identity at the level of the $16 \mathrm{~S}$ rRNA gene sequences, suggesting that the gene exchange mediated by ICE and the active distribution of ICE were ongoing.

The exogenous genes in clade II ICEs were mainly involved in fatty acid metabolism and (outer) membrane proteins, such as receptors, permeases, lipoproteins, phytochromes, and $\mathrm{Na}^{+} / \mathrm{H}^{+}$antiporters. These different types of ICEs with distinct foreign genes may have provided their own selective benefits under diverse environments to their hosts.

\section{CONCLUSION}

A comparison of 14 genomes with scattered distribution of AAPB revealed the gain and loss of phototrophic genes co-occurring in the evolutionary history of the genus Erythrobacter. The Erythrobacter genomes diverged into three separated groups with a large range of genome sizes. The ICEs might play significant roles in shaping or contributing to the large pan-genome of Erythrobacter. This study broadens our understanding of the phototrophic lifestyle evolutionary processes. With more novel species identified and whole genomes sequenced in this genus, future detailed analysis should further clarify the evolutionary history of phototrophy.

\section{AUTHOR CONTRIBUTIONS}

Conceived and designed the experiments: QZ, NJ, CC. Performed the experiments: QZ, WL, YL, CC. Analyzed the data: QZ, WL, YL, CC. Contributed reagents/materials/analysis tools: WL, YL. Wrote the paper: QZ, WL, YL, CC, NJ.

\section{ACKNOWLEDGMENTS}

This work was supported by the NSFC project (41306126), the 973 program (2013CB955700), the SOA project (GASI-0301-02-05), the Fundamental Research Funds for the Central Universities (2013121051), the NSFC (J1210050), the XMU Undergraduate Innovation and Entrepreneurship Training Programs (2014X0058) and the NSFC (31272697).

\section{SUPPLEMENTARY MATERIAL}

The Supplementary Material for this article can be found online at: http://journal.frontiersin.org/article/10.3389/fmicb. 2016.00984

Figure S1 | Sizes of the core (green) and pan-genomes (blue) of the Erythrobacter strains.

Figure S2 | Neighbor-joining phylogenetic trees based on VirB4 amino acid sequences. Bootstrap percentages from both neighbor-joining (above nodes) and maximum likelihood (below nodes) are shown.

Table S1 | Average Nucleotide Identity by pairwise genome comparison.

Table S2 | The upstream and downstream genes of PGCs in seven AAPB genoems.

Table S3 | Annotation for T4SS complex and T-DNA complex.

\section{REFERENCES}

Alvarez-Martinez, C. E., and Christie, P. J. (2009). Biological diversity of prokaryotic type IV secretion systems. Microbiol. Mol. Biol. Rev. 73, 775-808. doi: 10.1128/MMBR.00023-09

Anderson, C., Johnson, H., Caputo, N., Davis, R., Torpey, J., and Tebo, B. (2009). Mn (II) oxidation is catalyzed by heme peroxidases in "Aurantimonas manganoxydans" strain SI85-9A1 and Erythrobacter sp. strain SD-21. Appl. Environ. Microbiol. 75, 4130-4138. doi: 10.1128/AEM. 02890-08

Aylward, F. O., Mcdonald, B. R., Adams, S. M., Valenzuela, A., Schmidt, R. A., Goodwin, L. A., et al. (2013). Comparison of 26 sphingomonad genomes reveals diverse environmental adaptations and biodegradative capabilities. Appl. Environ. Microbiol. 79, 3724-3733. doi: 10.1128/AEM.00518-13

Aziz, R. K., Bartels, D., Best, A. A., Dejongh, M., Disz, T., Edwards, R. A., et al. (2008). The RAST Server: rapid annotations using subsystems technology. BMC Genomics 9:75. doi: 10.1186/1471-2164-9-75

Beatty, J. T. (1995). "Organization of photosynthesis gene transcripts," in Anoxygenic Photosynthetic Bacteria, eds R. E. Blankenship, M. T. Madigan, and C. E. Bauer (Springer Netherlands), 1209-1219. 
Béjà, O., Suzuki, M. T., Heidelberg, J. F., Nelson, W. C., Preston, C. M., Hamada, T., et al. (2002). Unsuspected diversity among marine aerobic anoxygenic phototrophs. Nature 415, 630-633. doi: 10.1038/415630a

Blankenship, R. E. (1992). Origin and early evolution of photosynthesis. Photosyn. Res. 33, 91-111. doi: 10.1007/BF00039173

Böltner, D., Macmahon, C., Pembroke, J. T., Strike, P., and Osborn, A. M. (2002). R391: a conjugative integrating mosaic comprised of phage, plasmid, and transposon elements. J. Bacteriol. 184, 5158-5169. doi: 10.1128/JB.184.18.51585169.2002

Burrus, V., Marrero, J., and Waldor, M. K. (2006). The current ICE age: biology and evolution of SXT-related integrating conjugative elements. Plasmid 55, 173-183. doi: 10.1016/j.plasmid.2006.01.001

Cho, J. C., Stapels, M. D., Morris, R. M., Vergin, K. L., Schwalbach, M. S., Givan, S. A., et al. (2007). Polyphyletic photosynthetic reaction centre genes in oligotrophic marine Gammaproteobacteria. Environ. Microbiol. 9, 1456-1463. doi: 10.1111/j.1462-2920.2007.01264.x

Ferrera, I., Borrego, C. M., Salazar, G., and Gasol, J. M. (2014). Marked seasonality of aerobic anoxygenic phototrophic bacteria in the coastal NW Mediterranean Sea as revealed by cell abundance, pigment concentration and pyrosequencing of pufM gene. Environ. Microbiol. 16, 2953-2965. doi: $10.1111 / 1462-2920.12278$

Fuchs, B. M., Spring, S., Teeling, H., Quast, C., Wulf, J., Schattenhofer, M., et al. (2007). Characterization of a marine gammaproteobacterium capable of aerobic anoxygenic photosynthesis. Proc. Natl. Acad. Sci. U.S.A. 104, 2891-2896. doi: 10.1073/pnas.0608046104

Gaillard, M., Pradervand, N., Minoia, M., Sentchilo, V., Johnson, D. R., and Van Der Meer, J. R. (2010). Transcriptome analysis of the mobile genome ICEclc in Pseudomonas knackmussii B13. BMC Microbiol. 10:153. doi: 10.1186/14712180-10-153

Guglielmini, J., Quintais, L., Garcillán-Barcia, M. P., De La Cruz, F., and Rocha, E. (2011). The repertoire of ICE in prokaryotes underscores the unity, diversity, and ubiquity of conjugation. PLoS Genet. 7:e1002222. doi: 10.1371/journal.pgen.1002222

Hubber, A., Vergunst, A. C., Sullivan, J. T., Hooykaas, P. J., and Ronson, C. W. (2004). Symbiotic phenotypes and translocated effector proteins of the Mesorhizobium loti strain R7A VirB/D4 type IV secretion system. Mol. Microbiol. 54, 561-574. doi: 10.1111/j.1365-2958.2004.04292.x

Jain, R., Rivera, M. C., and Lake, J. A. (1999). Horizontal gene transfer among genomes: the complexity hypothesis. Proc. Natl. Acad. Sci. U.S.A. 96, 3801-3806. doi: 10.1073/pnas.96.7.3801

Jiao, N., Zhang, F., and Hong, N. (2010). Significant roles of bacteriochlorophylla supplemental to chlorophylla in the ocean. ISME J. 4, 595-597. doi: 10.1038/ismej.2009.135

Jiao, N., Zhang, Y., Zeng, Y., Hong, N., Liu, R., Chen, F., et al. (2007). Distinct distribution pattern of abundance and diversity of aerobic anoxygenic phototrophic bacteria in the global ocean. Environ. Microbiol. 9, 3091-3099. doi: 10.1111/j.1462-2920.2007.01419.x

Jung, Y.-T., Park, S., Oh, T.-K., and Yoon, J.-H. (2012). Erythrobacter marinus sp. nov., isolated from seawater. Int. J. Syst. Evol. Microbiol. 62, 2050-2055. doi: 10.1099/ijs.0.034702-0

Kobližek, M., Béjà, O., Bidigare, R. R., Christensen, S., Benitez-Nelson, B., Vetriani, C., et al. (2003). Isolation and characterization of Erythrobacter sp. strains from the upper ocean. Arch. Microbiol. 180, 327-338. doi: 10.1007/s00203-00 3-0596-6

Koblížek, M., Janouškovec, J., Oborník, M., Johnson, J. H., Ferriera, S., and Falkowski, P. G. (2011). Genome sequence of the marine photoheterotrophic bacterium Erythrobacter sp. strain NAP1. J. Bacteriol. 193, 5881-5882. doi: 10.1128/JB.05845-11

Koblížek, M., Mašín, M., Ras, J., Poulton, A. J., and Prášil, O. (2007). Rapid growth rates of aerobic anoxygenic phototrophs in the ocean. Environ. Microbiol. 9, 2401-2406. doi: 10.1111/j.1462-2920.2007.01354.x

Kobližek, M., Zeng, Y., Horák, A., and Oborník, M. (2013). Regressive evolution of photosynthesis in the Roseobacter clade. Adv. Bot. Res. 66, 385-405. doi: 10.1016/B978-0-12-397923-0.00013-8

Kolber, Z. S., Gerald, F., Lang, A. S., Beatty, J. T., Blankenship, R. E., Vandover, C. L., et al. (2001). Contribution of aerobic photoheterotrophic bacteria to the carbon cycle in the ocean. Science 292, 2492-2495. doi: $10.1126 /$ science. 1059707
Koonin, E. V., Makarova, K. S., and Aravind, L. (2001). Horizontal gene transfer in prokaryotes: quantification and classification. Ann. Rev. Microbiol. 55, 709-742. doi: 10.1146/annurev.micro.55.1.709

Lagesen, K., Hallin, P., Rødland, E., StÃęrfeldt, H., Rognes, T., and Ussery, D. (2007). RNammer: consistent annotation of rRNA genes in genomic sequences. Nucleic Acids Res. 35, 3100-3108. doi: 10.1093/nar/gkm160

Lee, Y. S., Lee, D.-H., Kahng, H.-Y., Kim, E. M., and Jung, J. S. (2010). Erythrobacter gangjinensis sp. nov., a marine bacterium isolated from seawater. Int. J. Syst. Evol. Microbiol. 60, 1413-1417. doi: 10.1099/ijs.0.015743-0

Lei, X., Zhang, H., Chen, Y., Li, Y., Chen, Z., Lai, Q., et al. (2015). Erythrobacter luteus sp. nov., isolated from mangrove sediment. Int. J. Syst. Evol. Microbiol. 65, 2472-2478. doi: 10.1099/ijs.0.000283

Li, L., Stoeckert, C. J., and Roos, D. S. (2003). OrthoMCL: identification of ortholog groups for eukaryotic genomes. Genome Res. 13, 2178-2189. doi: 10.1101/gr.1224503

Lowe, T. M., and Eddy, S. R. (1997). tRNAscan-SE: a program for improved detection of transfer RNA genes in genomic sequence. Nucleic Acids Res. 25, 0955-0964. doi: 10.1093/nar/25.5.0955

Miyazaki, R., Bertelli, C., Benaglio, P., Canton, J., De Coi, N., Gharib, W. H., et al. (2015). Comparative genome analysis of Pseudomonas knackmussii B13, the first bacterium known to degrade chloroaromatic compounds. Environ. Microbiol. 17, 91-104. doi: 10.1111/1462-2920.12498

Nitschke, W., and Dracheva, S. M. (1995). "Reaction center associated cytochromes," in Anoxygenic Photosynthetic Bacteria, eds R. E. Blankenship, M. T. Madigan, and C. E. Bauer (Springer Netherlands), 775-805.

Oh, H.-M., Giovannoni, S. J., Ferriera, S., Johnson, J., and Cho, J.-C. (2009). Complete genome sequence of Erythrobacter litoralis HTCC2594. J. Bacteriol. 191, 2419-2420. doi: 10.1128/JB.00026-09

Poulin-Laprade, D., Matteau, D., Jacques, P., Rodrigue, S., and Burrus, V. (2015). Transfer activation of SXT/R391 integrative and conjugative elements: unraveling the SetCD regulon. Nucleic Acids Res. 43, 2045-2056. doi: 10.1093/nar/gkv071

Richter, M., and Rosselló-Móra, R. (2009). Shifting the genomic gold standard for the prokaryotic species definition. Proc. Natl. Acad. Sci. U.S.A. 106, 19126-19131. doi: 10.1073/pnas.0906412106

Ritchie, A. E., and Johnson, Z. I. (2012). Abundance and genetic diversity of aerobic anoxygenic phototrophic bacteria of coastal regions of the Pacific Ocean. Appl. Environ. Microbiol. 78, 2858-2866. doi: 10.1128/AEM.06268-11

Shiba, T., and Simidu, U. (1982). Erythrobacter longus gen. nov., sp. nov., an aerobic bacterium which contains bacteriochlorophyll $a$. Int. J. Syst. Bacteriol. 32, 211-217. doi: 10.1099/00207713-32-2-211

Spring, S., Lünsdorf, H., Fuchs, B. M., and Tindall, B. J. (2009). The photosynthetic apparatus and its regulation in the aerobic gammaproteobacterium Congregibacter litoralis gen. nov., sp. nov. PLoS ONE 4:e4866. doi: 10.1371/journal.pone.0004866

Stegman, M. R., Cottrell, M. T., and Kirchman, D. L. (2014). Leucine incorporation by aerobic anoxygenic phototrophic bacteria in the Delaware estuary. ISME J. 8, 2339-2348. doi: 10.1038/ismej.2014.75

Swingley, W. D., Blankenship, R. E., and Raymond, J. (2009). "Evolutionary relationships among purple photosynthetic bacteria and the origin of proteobacterial photosynthetic systems," in The Purple Phototrophic Bacteria, eds C. N. Hunter, F. Daldal, M. C. Thurnauer, and J. T. Beatty (Springer Netherlands), 17-29. doi: 10.1007/978-1-4020-8815-5_2

Tamura, K., Stecher, G., Peterson, D., Filipski, A., and Kumar, S. (2013). MEGA6: molecular evolutionary genetics analysis version 6.0. Mol. Biol. Evol. 30, 2725-2729. doi: 10.1093/molbev/mst197

Tettelin, H., Masignani, V., Cieslewicz, M. J., Donati, C., Medini, D., Ward, N. L., et al. (2005). Genome analysis of multiple pathogenic isolates of Streptococcus agalactiae: implications for the microbial "pan-genome". Proc. Natl. Acad. Sci. U.S.A. 102, 13950-13955. doi: 10.1073/pnas.05067 58102

Tonon, L. A. C., Moreira, A. P. B., and Thompson, F. (2014). "The family Erythrobacteraceae," in The Prokaryotes, eds E. Rosenberg, E. F. DeLong, S. Lory, E. Stackebrandt, and F. Thompson (Springer Berlin Heidelberg), 213-235. doi: 10.1007/978-3-642-30197-1_376

Verméglio, A., Nagashima, S., Alric, J., Arnoux, P., and Nagashima, K. V. (2012). Photo-induced electron transfer in intact cells of Rubrivivax gelatinosus mutants deleted in the RC-bound tetraheme cytochrome: insight into evolution 
of photosynthetic electron transport. Biochim. Biophys. Acta 1817, 689-696. doi: 10.1016/j.bbabio.2012.01.011

Wagner-Döbler, I., and Biebl, H. (2006). Environmental biology of the marine Roseobacter lineage. Annu. Rev. Microbiol. 60, 255-280. doi: 10.1146/annurev.micro.60.080805.142115

Wei, J., Mao, Y., Zheng, Q., Zhang, R., and Wang, Y.-N. (2013). Erythrobacter westpacificensis sp. nov., a marine bacterium isolated from the Western Pacific. Curr. Microbiol. 66, 385-390. doi: 10.1007/s00284-012-0287-0

Wozniak, R. A., Fouts, D. E., Spagnoletti, M., Colombo, M. M., Ceccarelli, D., Garriss, G., et al. (2009). Comparative ICE genomics: insights into the evolution of the SXT/R391 family of ICEs. PLoS Genet. 5:e1000786. doi: 10.1371/journal.pgen.1000786

Yaakop, A. S., Chan, C. S., Kahar, U. M., Ee, R., Chan, K.-G., and Goh, K. M. (2015). Draft genome sequence of Erythrobacter vulgaris strain O1, a glycosyl hydrolase-producing bacterium. Genome Announc. 3:e00457-15. doi: 10.1128/genomeA.00457-15

Yeo, H.-J., and Waksman, G. (2004). Unveiling molecular scaffolds of the type IV secretion system. J. Bacteriol. 186, 1919-1926. doi: 10.1128/JB.186.7.19191926.2004

Yurkov, V., and Csotonyi, J. T. (2009). "New light on aerobic anoxygenic phototrophs," in The Purple Phototrophic Bacteria, eds C. N. Hunter, F. Daldal, M. C. Thurnauer, and J. T. Beatty (Springer Netherlands), 31-55. doi: 10.1007/978-1-4020-8815-5_3

Yurkov, V., Stackebrandt, E., Holmes, A., Fuerst, J. A., Hugenholtz, P., Golecki, J., et al. (1994). Phylogenetic positions of novel aerobic, Bacteriochlorophyll acontaining bacteria and description of Roseococcus thiosulfatophilus gen. nov., sp. nov., Erythromicrobium ramosum gen. nov., sp. nov., and Erythrobacter litoralis sp. nov. Int. J. Syst. Bacteriol. 44, 427-434. doi: 10.1099/00207713-4 4-3-427

Yurkov, V. V., Krieger, S., Stackebrandt, E., and Beatty, J. T. (1999). Citromicrobium bathyomarinum, a novel aerobic bacterium isolated from deepsea hydrothermal vent plume waters that contains photosynthetic pigmentprotein complexes. J. Bacteriol. 181, 4517-4525.

Yutin, N., Suzuki, M. T., Teeling, H., Weber, M., Venter, J. C., Rusch, D. B., et al. (2007). Assessing diversity and biogeography of aerobic anoxygenic phototrophic bacteria in surface waters of the Atlantic and Pacific Oceans using the Global Ocean Sampling expedition metagenomes. Environ. Microbiol. 9, 1464-1475. doi: 10.1111/j.1462-2920.2007.01265.x
Zeng, Y., Feng, F., Medová, H., Dean, J., and Kobližek, M. (2014). Functional type 2 photosynthetic reaction centers found in the rare bacterial phylum Gemmatimonadetes. Proc. Natl. Acad. Sci. U.S.A. 111, 7795-7800. doi: $10.1073 /$ pnas. 1400295111

Zerbino, D. R., and Birney, E. (2008). Velvet: algorithms for de novo short read assembly using de Bruijn graphs. Genome Res. 18, 821-829. doi: 10.1101/gr.074492.107

Zheng, Q., Koblizek, M., Beatty, J. T., and Jiao, N. (2013). Evolutionary divergence of marine aerobic anoxygenic phototrophic Bacteria as seen from diverse organisations of their photosynthesis gene clusters. Adv. Bot. Res. 66, 359-383. doi: 10.1016/B978-0-12-397923-0.00012-6

Zheng, Q., Zhang, R., Fogg, P. C., Beatty, J. T., Wang, Y., and Jiao, N. (2012). Gain and loss of phototrophic genes revealed by comparison of two Citromicrobium bacterial genomes. PLoS ONE 7:e35790. doi: 10.1371/journal.pone. 0035790

Zheng, Q., Zhang, R., Koblížek, M., Boldareva, E. N., Yurkov, V., Yan, S., et al. (2011). Diverse arrangement of photosynthetic gene clusters in aerobic anoxygenic phototrophic bacteria. PLOS ONE 6:e25050. doi: 10.1371/journal.pone.0025050

Zheng, Q., Zhang, R., Xu, Y., White Iii, R. A., Wang, Y., Luo, T., et al. (2014). A marine inducible prophage vB_CibM-P1 isolated from the aerobic anoxygenic phototrophic bacterium Citromicrobium bathyomarinum JL354. Sci. Rep. 4, 4:7118. doi: $10.1038 /$ srep07118

Zhuang, L., Liu, Y., Wang, L., Wang, W., and Shao, Z. (2015). Erythrobacter atlanticus sp. nov., a bacterium from ocean sediment able to degrade polycyclic aromatic hydrocarbons. Int. J. Syst. Evol. Microbiol. 65, 3714-3719. doi: 10.1099/ijsem.0.000481

Conflict of Interest Statement: The authors declare that the research was conducted in the absence of any commercial or financial relationships that could be construed as a potential conflict of interest.

Copyright (c) 2016 Zheng, Lin, Liu, Chen and Jiao. This is an open-access article distributed under the terms of the Creative Commons Attribution License (CC BY). The use, distribution or reproduction in other forums is permitted, provided the original author(s) or licensor are credited and that the original publication in this journal is cited, in accordance with accepted academic practice. No use, distribution or reproduction is permitted which does not comply with these terms. 